\title{
REVISIONES
}

\section{La falacia de la conjunción y la contextualización en el autismo}

\author{
The conjunction fallacy and the contextualization in autism
}

A falácia da conjunção e contextualização no autismo

\author{
Miguel López \\ Departamento de Educación, Universidad de Los Lagos (Osorno, Chile). \\ Fono: (64) 333130. E-mail: m.lopez@ulagos.cl
}

\begin{abstract}
RESUMEN
Podemos interpretar, a partir de los resultados de algunos trabajos experimentales, que los estudiantes autistas no parecen utilizar heurísticos y que su pensamiento lógico-matemático es más riguroso que el del estudiante general. Morsanyi, Handley y Evans (2009) han presentado un estudio en el que cuestionan tal interpretación y explican que los estudiantes autistas manifiestan dificultades en sus procesos de contextualización de información. En este trabajo argumentamos que los resultados de la investigación de Morsanyi, Handley y Evans son cuestionables y que no demuestran categóricamente que los problemas de contextualización, y no su rigor lógico, sean la causa por la que los estudiantes autistas no utilizan ciertos heurísticos.
\end{abstract}

Palabras clave: autismo, contextualización, heurísticos, pensamiento lógico.

\begin{abstract}
By means of the outcomes of some experimental studies, we can interpret that autistic students do not seem to use heuristics. Furthermore, their logical-mathematical thinking is more rigorous than the general students' thinking. Morsanyi, Handley and Evans (2009) have presented a research that questions this interpretation and they have explained that autistic students have difficulties in their contextualization of information processes. In this paper, I argue that the outcomes of the research presented by Morsanyi, Handley and Evans can be questioned. They do not demonstrate categorically that the problems of contextualización, and not their logical rigor, are the reason for which the autistic students do not use certain heuristic.
\end{abstract}

Key words: autism, contextualization, heuristics, logical thinking.

\section{RESUMO}

Podemos interpretar, a partir dos resultados de alguns trabalhos experimentais, que os estudantes autistas não parecem utilizar heurísticos, e que seu pensamento lógico-matemático é mais rigoroso que o do estudante geral. Morsanyi, Handley e Evans (2009) apresentaram um estudo no qual questionam tal interpretação e explicam que os estudantes autistas manifestam dificuldades nos seus processos de contextualização de informação. Neste trabalho, argumentamos que os resultados da investigação de Morsanyi, Handley e Evans são questionáveis e que não demonstram categoricamente, que os problemas de contextualização, e não seu rigor lógico seja a causa pela qual os estudantes autistas não utilizam certos heurísticos.

Palavras-chave: autismo, contextualização, pensamento lógico, heurísticos. 


\section{INTRODUCCION}

No cabe duda de que el profesorado que tiene que atender a alumnado diagnosticado como perteneciente al espectro autista necesita conocer cuáles son exactamente sus singularidades cognitivas. Los estudios al respecto son diversos, pero algunos de ellos son particularmente interesantes, ya que pretenden llegar a conocer cómo funciona verdaderamente el razonamiento lógico-matemático en estos estudiantes. En este sentido, la investigación de Morsanyi, Handley y Evans (2009) parece de suma relevancia.

Morsanyi et al. (2009) analizan, entre otros aspectos, cómo responden los adolescentes autistas a un ejercicio bastante conocido en el área de la ciencia cognitiva: el problema de Linda (Tversky y Kahneman 1983). En este problema sucede que los sujetos experimentales que se enfrentan a él parecen incurrir en la denominada falacia de la conjunción, que consiste en que el individuo asigna a un acontecimiento simple una probabilidad menor que a un acontecimiento compuesto del que él forma parte. Este comportamiento es completamente opuesto a las leyes de la probabilidad y parece sugerir que los sujetos no entienden el significado exacto de la conjunción lógica ni operan mentalmente de acuerdo con las leyes probabilísticas, sino por medio de heurísticos o sesgos.

Para comprender verdaderamente en qué consiste la falacia de la conjunción y cuáles son sus repercusiones en el ámbito cognitivo, puede ser necesario conocer las características generales del problema de Linda. En él, se le cuenta al individuo cómo era Linda, una mujer de 31 años en el momento presente, cuando era estudiante. Así, se le dice que estudió Filosofía y que luchaba contra la discriminación y contra diversas formas de injusticia social. Finalizada la descripción de Linda, se le presentan al sujeto ocho posibilidades de situaciones de Linda en la actualidad y se le solicita que las ordene de mayor a menor probabilidad. La mayor parte de esas posibilidades funcionan como distractores, pues las cruciales parecen ser dos:

A. Linda trabaja en la caja de un banco.

B. Linda trabaja en la caja de un banco y milita en el movimiento feminista.

Altos porcentajes de participantes consideran a B más probable que A, lo cual, como hemos indicado, atenta contra las leyes más elementales de la teoría de la probabilidad, ya que significa atribuir a un suceso compuesto (B) que incluye una conjunción ("y") una probabilidad más elevada que a un suceso simple (A). Éste es el motivo por el que se conoce a este fenómeno con el nombre de la falacia de la conjunción.

A partir de estos hechos, se ha interpretado que la población general no necesariamente sigue los principios lógico-matemáticos, sino que se ve condicionada por una serie de heurísticos relacionados con la representatividad de los conceptos (Tversky y Kahneman 1983). Así, puede decirse que en la mente de los sujetos no rigen las leyes probabilísticas, sino que desempeña un papel más relevante el hecho de que, si tenemos en cuenta las características de Linda expuestas en el problema que lleva su nombre, el enunciado B representa mucho mejor la posible situación de Linda en el presente.

No obstante, otro hecho sorprendente parece acompañar a este ejercicio de razonamiento: al plantearse a estudiantes con autismo, éstos ofrecen, significativamente, resultados más correctos que la población general. Según parece, no se ven influidos por la descripción de Linda y aplican las reglas lógico-matemáticas con mayor rigor que 
estudiantes no autistas, por lo que, aparentemente, no son afectados, en un porcentaje considerable, por la falacia de la conjunción. Un estudio de esta índole es precisamente el que podemos encontrar en el ya citado trabajo de Morsanyi et al. (2009).

Como sabemos, generalmente entendemos por autismo un desorden que va acompañado de importantes dificultades en el ámbito de la interacción social y la comunicación, así como de conductas repetitivas, estereotipadas y restringidas e intereses bastante limitados (DSM-IV 1994). En algunos trabajos, se ha insistido, además, en la reducida flexibilidad mental de los autistas (por ejemplo, Hill 2004) y en su incapacidad para modificar una acción o un pensamiento cuando el contexto o la situación cambian (por ejemplo, Pijnacker, Geurts, Van Lambalgen, Kan, Buitelaar y Hagoort 2009).

Ante tales descripciones del autismo, Morsanyi et al. (2009) consideran que la mejor ejecución de los adolescentes autistas que participan en su experimento de su versión del problema de Linda no se debe a que posean capacidades de pensamiento lógicomatemático superiores a las de los adolescentes no autistas. En su opinión, los resultados que se obtienen obedecen a las dificultades que presentan los autistas para contextualizar información, es decir, para, en el caso del problema que nos ocupa, relacionar la descripción de Linda con las posibilidades ofrecidas a los participantes.

Sin embargo, desde nuestro punto de vista, las conclusiones de Morsanyi et al. (2009) son discutibles, ya que las respuestas de sus sujetos experimentales son susceptibles de interpretaciones diversas. Existe la posibilidad de que los sujetos generales no cometan realmente la falacia de la conjunción en el problema de Linda y, por tanto, de que no se vean condicionados, como defienden Tversky y Kahneman (1983), por heurísticos relativos a la representatividad de los conceptos. Los extraños resultados de este ejercicio bien pueden deberse al procesamiento que los participantes realizan de los enunciados que describen las distintas opciones para Linda en el momento presente. Si esto es así, la investigación de Morsanyi et al. (2009) no demuestra verdaderamente que los estudiantes autistas tengan problemas con la contextualización de los mensajes lingüísticos y que no destaquen por sus capacidades lógico-matemáticas. Y es que puede que lo único que suceda sea que los alumnos con autismo procesan la información de un modo diferente al estudiante general. En concreto, nosotros sostenemos que los estudiantes autistas manifiestan tendencias a entender los enunciados de una manera más literal que el alumnado general y, en lo que sigue, vamos a intentar defender esta tesis. No obstante, para ello, es fundamental exponer, en primer lugar, cuáles son las versiones del problema de Linda con las que trabajan Morsanyi et al. (2009) y cómo interpretan los datos porcentuales que arroja su experimento.

\section{EL PROBLEMA DE LINDA Y EL AUTISMO}

Morsanyi et al. (2009) presentan, en realidad, dos tipos de versiones del problema de Linda con el propósito de comparar sus resultados. Los ejercicios que presentan como correspondientes a uno de estos tipos, denominado Conflict (Conflicto), se ajustan a las características generales del problema de Linda de Tversky y Kahneman (1983). Un ejemplo de problema representativo de las versiones Conflict es éste:

"Sue is a very intelligent woman, who works in a hospital. She wears glasses and a green uniform. Her bookshelves in her office are full of medical books. Mark the following statements with a number 1 to 4 according to how likely they are (1: most likely, 4: least likely). 


Sue is a plumber.
Sue is a doctor.
Sue is a doctor and a mechanic.
Sue is a mechanic" (Morsanyi et al., 2009: 76).

Es evidente que lo que se trata de comprobar con este problema es si los individuos consideran la alternativa Sue es una doctora y una mecánica más probable que la opción Sue es una mecánica, lo cual significaría incurrir en la falacia de la conjunción y, por tanto, asignar a un acontecimiento compuesto una probabilidad mayor que a un acontecimiento simple, acontecimiento, este último, que forma parte del acontecimiento compuesto.

Morsanyi et al. (2009) aplicaron tareas de esta índole a adolescentes diagnosticados con autismo y a un grupo de control constituido por adolescentes sin problemas de desarrollo detectados y, sorprendentemente, hallaron porcentajes más bajos de autistas que cometían la falacia de la conjunción. Mientras que el grupo de control caía en esta falacia en un $88 \%$ de casos, el grupo de autistas lo hacía sólo en un $72 \%$ de ocasiones. Es obvio que, en una primera intuición, podemos pensar que estos resultados demuestran que los estudiantes autistas disponen de mayores potencialidades lógico-matemáticas que la población general, potencialidades que deben ser tenidas en cuenta por los docentes y que podrían ser aprovechadas para un mejor aprendizaje de este tipo de alumnado.

Sin embargo, Morsanyi et al. (2009) no dedujeron ninguna conclusión en este sentido, ya que su segundo tipo de versiones del problema, las versiones Non-conflict (No conflicto), proporcionaron otra información que, desde su punto de vista, no era menos relevante. Los problemas que ellos plantearon como versiones Non-conflict aparentemente conservan la estructura inicial del problema de Linda de Tversky y Kahneman (1983), pero, en realidad, en sus instrucciones hay una diferencia esencial: la alternativa con un suceso compuesto que podría considerarse como más probable que un suceso simple no incluye un concepto representativo de la persona descrita, es decir, si pensamos en la versión del problema de Linda descrita en la introducción, las opciones con acontecimientos compuestos no presentan características que podamos relacionar directamente con la descripción de Linda. Un ejemplo de este tipo de problema que nos ofrecen Morsanyi et al. (2009) es el siguiente:

"Brian has a studio, where he works alone. He is a very creative man, and he likes to experiment with colours. He takes his work to exhibitions, and sells some of them too. Mark the following statements with a number 1 to 4 according to how likely they are (1: most likely, 4: least likely).

Brian is an aerobics instructor.

Brian es a painter.

1 Sue es una mujer muy inteligente, que trabaja en un hospital. Utiliza lentes y un uniforme verde. Los estantes de su oficina están llenos de libros de medicina. Marca las siguientes afirmaciones con un número del 1 al 4 según la probabilidad que posean (1: la más probable, 4: la menos probable).

Sue es una fontanera.

Sue es una doctora.

Sue es una doctora y una mecánica.

Sue es una mecánica. 
Brian is an aerobics instructor and an accountant.

Brian is an accountant"2 (Morsanyi et al. 2009: 76).

Como podemos apreciar en este ejemplo, la única alternativa que describe un suceso compuesto, Brian es un instructor de aerobic y un contador, no incluye ninguna característica claramente representativa del perfil de Brian. Lo previsible es que, ante un ejercicio como éste, los sujetos experimentales no consideren tal opción como más probable que cualquiera de las demás y que, por consiguiente, los porcentajes de individuos que cometen la falacia de la conjunción se vean significativamente reducidos. Ello, efectivamente, fue lo que ocurrió en el grupo de control, donde el $88 \%$ de errores se transformó en un 56\%. El dato clave residía, a juicio de Morsanyi et al. (2009), en que, aunque en el grupo de autistas el $72 \%$ de casos en los que se cometió la falacia de la conjunción descendió hasta un $65 \%$, tal disminución no reflejó una reducción estadísticamente significativa.

Así, Morsanyi et al. (2009) concluyen que los autistas sólo son aparentemente más sensibles a la conjunción lógica que la población general, pues, a su juicio, su ejecución de las versiones Non-conflict no nos autoriza a hablar de una mayor capacidad lógica en ellos. De hecho, Morsanyi et al. (2009) sostienen que los sujetos diagnosticados con autismo cometen menos errores lógicos en las versiones Conflict no porque razonen mejor, sino porque presentan problemas con la contextualización de la información o, dicho de otro modo, porque para ellos es más complejo relacionar las características que aparecen en las opciones a jerarquizar en función de su grado de probabilidad con la descripción del personaje protagonista narrada en las instrucciones.

No obstante, nosotros pensamos que, si tenemos en cuenta algunos trabajos presentados en el ámbito de la ciencia cognitiva, no parece tan evidente que las dificultades que los autistas manifiestan en las versiones Non-conflict propuestas por Morsanyi et al. (2009) sean el resultado de sus problemas para contextualizar. Podemos, de esta manera, analizar un planteamiento que no interpreta las respuestas de los sujetos en las versiones iniciales del problema de Linda de Tversky y Kahneman (1983) como el producto del uso del heurístico de la representatividad, sino como la consecuencia de procesamientos de la información contenida en las instrucciones distintos a los que el investigador prevé. A partir de este enfoque, se tornan, como vamos a apreciar, bastante dudosas las tesis de Morsanyi et al. (2009).

\section{EL EFECTO DE LAS REPRESENTACIONES MENTALES EN EL PROBLEMA DE LINDA}

El trabajo al que nos referimos no es otro que el que aparece en López Astorga (2009). En dicho trabajo, se analizan diversas versiones del problema de Linda, no sólo

2 Brian tiene un estudio, donde trabaja solo. Es un hombre muy creativo, y le gusta experimentar con colores. Lleva sus trabajos a exhibiciones y también vende algunos de ellos. Marca las siguientes afirmaciones con un número del 1 al 4 según la probabilidad que posean (1: la más probable, 4: la menos probable). Brian es un instructor de aerobic. Brian es un pintor. Brian es un instructor de aerobic y un contador. Brian es un contador. 
las diferentes que aparecen en Tversky y Kahneman (1983), sino también otras revisadas en distintas investigaciones, como, por ejemplo, en Díaz (2005) o en Fiedler (1988).

Entre otras tesis, en López Astorga (2009) se argumenta que los sujetos experimentales que se enfrentan al problema de Linda no utilizan ningún heurístico mental para ofrecer su ordenación de las alternativas según la probabilidad correspondiente a cada una de ellas. Lo que sucede en el citado problema es que los sujetos no procesan el acontecimiento simple al que consideran menos probable que un acontecimiento compuesto como un verdadero acontecimiento simple, sino como otro también compuesto. De esta manera, la falacia de la conjunción desaparece, ya que no se piensa que un suceso compuesto sea más probable que otro simple, sino, sencillamente, que un suceso compuesto es más probable que otro suceso compuesto.

En la versión inicial que comentamos en la introducción, esto significaría que los sujetos interpretan la opción A no como Linda trabaja en la caja de un banco, sino como Linda trabaja en la caja de un banco y no milita en el movimiento feminista, entendiendo así que cada una de las alternativas representa una definición completa de Linda y excluyente con respecto a todas las demás. Ciertamente, en sentido estricto, la opción A no implica que Linda no milite en el movimiento feminista. Realmente, deja la posibilidad abierta y permite tanto que Linda milite en tal movimiento como que no lo haga. No obstante, el participante puede entender que A y B son dos posibilidades incompatibles y que A supone, necesariamente, la no militancia de Linda en el movimiento feminista. Desde este punto de vista, según se deriva de los argumentos expuestos en López Astorga (2009), los sujetos experimentales no incurren en ninguna falacia, pues únicamente juzgan que un acontecimiento compuesto es más probable que otro que es igualmente compuesto.

Si aceptamos estas tesis, los resultados de Morsanyi et al. (2009) pueden ser interpretados desde otra perspectiva. La mejor ejecución de los autistas en sus versiones Conflict sólo implicaría que algunos autistas procesan la información presente en las alternativas de un modo más literal y que no vinculan los acontecimientos simples con otros acontecimientos simples negados. En cualquier caso, hemos de tener en cuenta que, si el enfoque planteado en López Astorga (2009) es correcto, hay que reconocer que los adolescentes autistas que se enfrentaron a las versiones Conflict de Morsanyi et al. (2009) resolvieron mejor el problema, ya que, al fin y al cabo, entendieron las instrucciones como había que hacerlo y no procesaron la opción Sue es una mecánica como Sue es una mecánica y no una doctora, sino que comprendieron que dicha opción no eliminaba la posibilidad de que Sue, además de ser mecánica, fuera doctora. Indiscutiblemente, si ésta fue la actuación del individuo con autismo, redundó en un razonamiento lógicomatemático más riguroso y eficaz.

De todos modos, la explicación ofrecida en López Astorga (2009) debería servir también para interpretar los resultados de las versiones Non-conflict de Morsanyi et al. (2009). Nosotros creemos que no es difícil acometer tal tarea desde el marco establecido en López Astorga (2009). Recordemos que lo único que ocurrió en las versiones Non-conflict es que, de una cantidad de errores de un $72 \%$ en las versiones Conflict, se descendió a un $65 \%$. Un porcentaje de un $72 \%$ es, claro está, un porcentaje elevado (a pesar de ser menor que el del grupo de control) y, por consiguiente, nos muestra que, aunque entre los individuos con autismo existe una mayor tendencia a interpretar los mensajes lingüísticos de un modo más literal, buena parte de ellos también comete la 
falacia de la conjunción. No debe resultarnos extraño, de este modo, que en la versión Non-conflict se continúe incurriendo en tal error y que se siga completando la información presentada en las opciones con características negadas.

Con respecto a esto último, hemos de decir que no sería poco común que un adolescente, a partir de una experiencia real, ya sea ésta próxima o lejana, o de un ejemplo procedente de la literatura de ficción o del mundo cinematográfico, estableciera relaciones entre ser pintor, ser instructor de aerobic y ser contador. En este sentido, debemos ser cautos y prudentes ante los resultados de versiones del problema de Linda como las Non-conflict que proponen Morsanyi et al. (2009), pues, en realidad, en vez de estar informándonos sobre las habilidades lógicas de los individuos que las realizan (sean éstos autistas o no), pueden estar reflejando solamente las representaciones mentales de los sujetos, las conexiones que establecen entre esas representaciones y otras y los vínculos que suponen entre diferentes conceptos e ideas. Probablemente, no existen dos individuos exactamente iguales en cuanto a sus características idiosincrásicas y, por esta razón, difícilmente podemos hallar dos personas con autismo que reaccionen de manera idéntica ante problemas de esta índole. Y es que, como hemos dicho, es importante no olvidar que, aunque se observa una clara mejora de los porcentajes de respuesta correcta en el problema de Linda cuando lo ejecutan participantes autistas, muchos sujetos con autismo no lo resuelven adecuadamente.

\section{CONTEXTUALIZACION Y AUTISMO}

Pero Morsanyi et al. (2009) podrían objetar contra la argumentación que acabamos de exponer que incluyen en su trabajo otros experimentos que apoyan sus tesis y que evidencian que la óptima ejecución de los autistas en el problema de Linda es sólo aparente, ya que su conducta intelectual es únicamente el resultado de sus dificultades para contextualizar información.

Sin embargo, nosotros también somos críticos con respecto a las demás condiciones experimentales de Morsanyi et al. (2009), las cuales versan, fundamentalmente, sobre el problema de los ingenieros y los abogados (Kahneman y Tversky 1973). En las versiones iniciales de este problema, se les habla a los participantes de, por ejemplo, 30 ingenieros y 70 abogados y se les describe a una persona que se incluye en este grupo de 100 individuos. Así, se les pregunta si creen que es un ingeniero o un abogado. Normalmente, se observa que los sujetos experimentales se guían más por la descripción de la persona que por la proporción de ingenieros y abogados (claramente, en este caso, a favor de los abogados) que se establece en las instrucciones.

Como decimos, intentando evidenciar lo complicado que puede llegar a ser para un autista contextualizar, Morsanyi et al. (2009) presentan cuatro tipos de versiones de este problema tanto a su grupo de adolescentes autistas como a su grupo de control, pero, en nuestra opinión, a pesar de la interpretación que hacen de sus resultados, la ejecución de los participantes en estas versiones, lejos de confirmar sus tesis, las cuestionan aún más. En primer lugar, sólo en uno de los cuatro tipos de versiones se observa un comportamiento significativamente diferente entre los adolescentes con autismo y los adolescentes sin tal diagnóstico. Por otra parte, algunas de las versiones que resuelven 
de modo semejante ambos grupos requieren, sin lugar a dudas, contextualizar la información que se presenta.

Morsanyi et al. (2009) nos reproducen un ejemplo del tipo de versiones en el que se aprecian diferencias evidentes. Tal ejemplo es el siguiente:

"Laura is a member of a sailing club and she also sings in a choir. She has 20 friends in the sailing club and 4 friends in the choir. Now she's going to Spain with a friend. Do you think it is more likely that she goes to Spain with somebody from the sailing club or with somebody from the choir?

It is more likely that it's somebody from the choir.

It is more likely that it's somebody from the sailing club.

Both are equally likely"3 (Morsanyi et al., 2009: 76).

Según Morsanyi et al. (2009), el grupo de control se inclinó en este ejercicio por las descripciones con significado social presentes en él, es decir, influyó en su decisión el que el amigo perteneciera al coro o al club de vela, mientras que los adolescentes autistas se centraron más en el número de amigos que Laura tiene en cada uno de los dos grupos. Este dato es considerado por Morsanyi y sus colaboradores como una prueba más de la complejidad que reviste la acción de contextualizar para la mente autista. Empero, tenemos que objetar que en las situaciones descritas en el problema, ser miembro de un coro o pertenecer a un club de vela, no hay verdaderamente ningún elemento decisivo y objetivo que pueda vincularse con el deseo de visitar España. Por tanto, desde nuestra óptica, no contextualizar en un ejercicio como éste es más adecuado que sí hacerlo, lo que sugiere que los resultados de una prueba de esta índole nada pueden decirnos sobre la capacidad de contextualizar información que tiene el individuo que lo realiza.

Otro tipo de versiones que proponen Morsanyi et al. (2009), en cambio, sí que implica necesariamente habilidad para la contextualización. Lo curioso es que en este tipo el grupo de adolescentes autistas no manifiesta un patrón de respuesta diferente al del grupo de control (como hemos apuntado, sólo en las versiones semejantes al problema de Laura se observan diferencias). Morsanyi et al. (2009) exponen un ejemplo de esta clase de versiones con estos términos:

"A group of tourists visit the Eiffel tower in Paris. There are 15 old people in the group and 3 young ones. The tourists can choose between taking the lift or climbing up the tower (which takes half an hour). Only one person wants to climb up the tower. Do you think it is more likely that it's an old person, or that it's a young one?

It is more likely that it's a young person.

It is more likely that it's an old person.

Both are equally likely"4 (Morsanyi et al., 2009: 76).

3 Laura es miembro de un club de vela y también canta en un coro. Tiene 20 amigos en el club de vela y 4 amigos en el coro. Ahora ella va a ir a España con un amigo. ¿Crees que es más probable que vaya a España con alguien del club de vela o con alguien del coro? Es más probable que sea alguien del coro. Es más probable que sea alguien del club de vela. Ambos son igualmente probables.

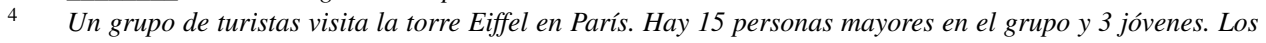


Es indiscutible que en problemas como éste la contextualización es necesaria, ya que es preciso atender a la probabilidad de que una persona con una edad determinada decida realizar un esfuerzo físico considerable. Lo llamativo aquí es que, como hemos mencionado, ambos grupos, el representante de los autistas y el de la población general, se inclinan por la respuesta obvia, sin poderse establecer importantes diferencias. Esto, ya lo hemos indicado, lejos de poder ser un apoyo para Morsanyi et al. (2009), lo es para nuestras tesis, pues muestra que los estudiantes con autismo pueden llegar a ser capaces de contextualizar en buena medida.

Morsanyi et al. (2009) recurren, igualmente, a otros argumentos. Por ejemplo, nos cuentan que sus sujetos realizaron una prueba para medir su memoria operativa verbal tanto en su dimensión de procesamiento como en la de almacenamiento, basada en el trabajo de Handley, Capon, Beveridge, Dennis y Evans (2004), y que encontraron correlaciones claras entre las respuestas de ambos grupos y su capacidad de memoria operativa. No obstante, para nosotros, el que tales análisis revelen que los estudiantes autistas tienen que emplear un mayor esfuerzo que los estudiantes generales para ejecutar algunas tareas carece de importancia si el resultado de su labor en esas tareas es el mismo o, como hemos notado en algunos casos, mejor que el de los estudiantes generales en esas mismas tareas. Datos de este tipo sólo nos recuerdan que, ante alumnos diferentes, tenemos que utilizar una metodología distinta, y no nos prueban en absoluto que los autistas no puedan realizar una actividad determinada. Sin duda, esta es una información de suma relevancia para los docentes que atienden a alumnado con autismo, pero no nos muestra, de ningún modo, que tal alumnado no pueda enfrentarse con éxito a ciertos problemas o ejercicios.

\section{LA TEORIA DUAL DE RAZONAMIENTO: EL AUTISMO Y EL SISTEMA 1}

Consideramos que lo expuesto hasta ahora es suficiente para, al menos, hacernos dudar de las conclusiones que extraen Morsanyi et al. (2009) a partir de sus resultados experimentales. Como afirma Mario Bunge, "sólo la teoría puede convertir ciertos datos en evidencias relativas a objetos inobservables" (Bunge 1985: 755). Los mismos hechos pueden vincularse con teorías e hipótesis radicalmente opuestas, y ello lo demuestran, entendemos, las argumentaciones que hemos presentado en las páginas previas. Morsanyi et al. (2009) piensan que sus resultados prueban que los adolescentes con autismo manifiestan problemas en sus procesos de contextualización. Nosotros, sin embargo, interpretamos que esos mismos datos apoyan nuestra hipótesis relativa a que los estudiantes autistas se ciñen a la información con mayor literalidad, lo que les beneficia en lo referente al razonamiento lógico-matemático. Esto no significa que defendamos necesariamente que la mente autista está dotada con capacidades lógicas más óptimas, sino que recurre a la

turistas pueden elegir entre tomar el ascensor o subir a pie la torre (lo cual toma media hora). Sólo una persona quiere subir a pie la torre. ¿Crees que es más probable que sea una persona mayor o que sea una joven?

Es más probable que sea una persona joven.

Es más probable que sea una persona mayor.

Ambos son igual de probables. 
utilización de tales capacidades con mayor frecuente que la mente general, la cual, en muy diversas ocasiones, opera por medio de heurísticos.

Una crítica contra nuestra propuesta bien puede ser que se trata de una opinión tan válida, y tan discutible, como la de Morsanyi et al. (2009). No obstante, creemos que las últimas investigaciones en el ámbito de la ciencia cognitiva avalan nuestro enfoque y que nuestras hipótesis son más cercanas a tales investigaciones que las de Morsanyi et al. (2009).

En este sentido, podemos decir que una teoría cognitiva relativamente reciente que sería consistente con nuestro planteamiento es la teoría dual del razonamiento (véase, por ejemplo, Stanovich, 1999, o Evans, 2008). Según esta teoría, los seres humanos contamos con dos sistemas diferentes en nuestra mente: el Sistema 1 y el Sistema $2^{5}$. El Sistema 1 es inconsciente, muy rápido y está regido por heurísticos, mientras que el Sistema 2, por el contrario, se encuentra relacionado con el razonamiento analítico, es lento y es posible tener conciencia de él.

De esta manera, podemos pensar que, mientras en el Sistema 1 operan heurísticos como el de la representatividad defendido por Tversky y Kahneman (1983), el Sistema 2 tiene que ver directamente con el razonamiento lógico y matemático. Parece, además, que, si no todos, muchos de los heurísticos del Sistema 1 son aprendidos y adquiridos por la práctica (Reyna 2004). Así, a partir del trabajo de Inglis y Simpson (2006) y de la interpretación del mismo que aparece en López Astorga (2009b), podemos explicar, por ejemplo, por qué los grandes maestros del ajedrez no perciben el tablero de la misma forma que los aficionados y pueden enfrentar varias partidas al mismo tiempo, pues operan con heurísticos inconscientes, intuitivos, aprendidos con el ejercicio y la práctica y que se hallan en su Sistema 1.

Sin embargo, lo que a nosotros nos interesa en este trabajo es la tesis de Inglis y Simpson (2006) relativa a que los extraños resultados de la población general en el problema de Linda son debidos a que los sujetos resuelven este problema utilizando el Sistema 1, y no el Sistema 2. Disponemos de heurísticos mentales, instalados en el Sistema 1, que, atendiendo a la versión del problema que presentamos en la introducción, relacionan la descripción de Linda con su militancia en el movimiento feminista. De hecho, Inglis y Simpson (2006) están convencidos de que los resultados habituales del problema de Linda constituyen una evidencia empírica para la teoría dual del razonamiento.

El caso es que, si los argumentos de Inglis y Simpson (2006) son válidos, lo que les sucede a los estudiantes autistas que ordenan correctamente las opciones que se les presentan en las versiones del problema de Linda de Morsanyi et al. (2009) es, según interpretamos, simplemente, que no operan con su Sistema 1, sino que, directamente, recurren a su Sistema 2. Esto supone que razonan lógicamente en el problema, lo cual no es lo que hacen los adolescentes generales, pues éstos se limitan a utilizar los heurísticos aprendidos de su Sistema 1.

Así, la lectura literal que realizan los sujetos diagnosticados con autismo de las instrucciones de los problemas les conduce a emplear capacidades lógico-matemáticas y analíticas características del Sistema 2, lo que, sin duda, supone un importante apoyo para nuestra tesis referente a que el estudiante autista emplea el razonamiento lógico con más frecuencia que la población general.

5 Esta terminología fue introducida, según parece y hasta donde sabemos, por Stanovich (1999). 
Pero una importante consecuencia se deriva de nuestro planteamiento: ¿es, por tanto, el Sistema 1 el que resulta deficitario en el estudiante autista? No necesariamente es así. Como hemos indicado, según Reyna (2004), muchos de los heurísticos con los que contamos se adquieren y se desarrollan con la práctica. Sabemos, y así se atestigua, ya lo dijimos, en DSM-IV (1994), que el autismo va acompañado de una manifestación de intereses muy particulares y circunscritos a temáticas bastante concretas. Posiblemente, los estudiantes autistas disponen de heurísticos en sus áreas de interés, y no en todos los ámbitos en los que podemos apreciarlos en la población general. En cualquier caso, el que no cuenten con ellos en algunas áreas no significa forzosamente que no puedan adquirirlos, sino únicamente que esas áreas no son de su preferencia.

Tenemos, por consiguiente, que, ante la carencia de heurísticos, los estudiantes autistas pueden emplear en más ocasiones que el alumno general el razonamiento lógico. Obviamente, ésta es otra información relevante para el profesorado que trabaja con estudiantes diagnosticados con autismo, pues, además de ayudarle a comprender de un modo más profundo a sus alumnos, puede indicarle cuáles pueden ser las estrategias metodológicas más adecuadas para utilizar en su práctica docente. Del mismo modo, le revela que el estudiante autista puede tener potencialidades nada desdeñables que no deben ser, bajo ningún concepto, ignoradas.

\section{CONCLUSIONES}

Quizás, la mente autista continúa siendo uno de los grandes enigmas para la psicología en el momento presente. Sin embargo, el interesante y necesario propósito de poner en práctica proyectos educativos inclusivos e integradores nos impone el reto de encontrar, con cierta celeridad, los recursos metodológicos más apropiados para las características idiosincrásicas de este colectivo. En nuestra búsqueda, empero, debemos tener muy presente que los hallazgos experimentales obtenidos en investigaciones diversas pueden ser interpretables en distintas direcciones y en función de diferentes marcos teóricos.

Morsanyi y sus colaboradores parecen pensar que los autistas sólo realizan tareas intelectuales de una manera más adecuada que la población general aparentemente. En su opinión, bajo su buena ejecución en problemas como los analizados en estas páginas subyacen importantes déficits relacionados con las destrezas necesarias para el procesamiento de información contextualizada. Además, desde su punto de vista, muchos de los logros de los sujetos autistas requieren altos niveles de esfuerzo cognitivo, niveles a los que no precisan llegar los individuos generales.

Nuestra óptica es muy diferente e interpretamos sus mismos resultados desde otra perspectiva, ya que, como se ha tornado evidente en estas páginas, partimos de supuestos muy distintos. A nuestro juicio, lo único que nos revelan sus experimentos es que los autistas presentan una tendencia mayor a la interpretación literal de los textos y de las instrucciones de los problemas, lo que los conduce, en muchas situaciones, a la eficacia intelectual. Por supuesto, ésta no es una constante en todos los individuos con autismo, lo cual explica las respuestas del grupo con autismo en la condición experimental Nonconflict de Morsanyi et al. (2009), pero, entre los sujetos autistas, se observa una mayor frecuencia de este modo de proceder. 
Para ningún docente debe ser un obstáculo el hecho de que un alumno necesite invertir mayor esfuerzo para ejecutar un ejercicio. Más bien, al contrario, debe suponer un incentivo para apoyar y atender individualizadamente al alumno en tales circunstancias. Al fin y al cabo, si los resultados son positivos, pueden no ser tan trascendentes ni el tiempo necesitado ni las energías requeridas para lograrlos.

Por otra parte, el alumnado con autismo parece usar en un número mayor de ocasiones que el alumnado no autista sus capacidades lógico-matemáticas. Éste es, sin duda, un punto controvertido y que puede ser objeto de intensas discusiones entre los teóricos y los investigadores, pero la teoría dual de razonamiento, una de las teorías que en la actualidad son aceptadas casi unánimemente por la mayor parte de los autores más reconocidos en el ámbito de la ciencia cognitiva, parece demostrar que es así. Por tanto, el profesorado responsable de alumnos autistas no debería ser ajeno a esta posibilidad, sino centrarse en ella y valorar hasta qué punto sus actividades pueden ser las más pertinentes para evaluar a sus estudiantes y a sus capacidades y pontencialidades. Es posible que el estudiante con autismo no sea más lógico que el estudiante sin autismo, pero también lo es, como decimos, que el autista recurra a la lógica en más situaciones que el no autista. Quizás, eso es lo que lo hace singular. Puede que su capacidad lógica no sea mayor, pero también es perfectamente admisible que use de manera más continua tal capacidad.

Tenemos que admitir, igualmente, que las pretensiones de Morsanyi et al. (2009) son legítimas y que, debido a todos los aspectos que aún desconocemos del autismo, cabe también la posibilidad de que a los autistas les resulten complejos sus procesos de contextualización. No obstante, con respecto a esto, son dos las afirmaciones que tenemos que expresar. En primer lugar, el trabajo de Morsanyi et al. (2009) no demuestra definitivamente que sea así y, en segundo lugar, hay que tener claro que manifestar problemas en la realización de ciertos procesos no significa no poder realizarlos.

En cualquier caso, la teoría dual de razonamiento parece aportarnos interesantes caminos de investigación para la temática que nos ha ocupado en estas páginas, pues la carencia de heurísticos en el Sistema 1 de la mente autista es perfectamente compatible con su débil interacción social y sus problemas de comunicación, los cuales pueden haber dificultado la adquisición o el ejercicio de tales heurísticos. Así, podría decirse que las capacidades lógico-matemáticas de los autistas pueden estar intactas y ser utilizadas a pleno rendimiento y que, sin embargo, la particularidad idiosincrásica de los individuos con autismo parece residir en el hecho de que, a causa de una dinámica social limitada, no se observan en ellos algunos modos intuitivos de proceder intelectualmente frecuentes en la población general.

\section{REFERENCIAS BIBLIOGRAFICAS}

Bunge, M. (1985). La investigación científica. Ariel. Barcelona.

Díaz, C. (2005). Evaluación de la falacia de la conjunción en estudiantes universitarios. Suma 48: $45-50$.

DSM-IV (1994). Diagnostic and statistical manual of mental disorders $\left(4^{\text {th }}\right.$ ed.). American Psychiatric Association. Washington, DC.

Evans, J. St. B.T. (2008). Dual-processing accounts of reasoning, judgement, and social cognition. Annual Review of Psychology 59: 255-278. 
Fiedler, K. (1988). The dependence of the conjunction fallacy on subtle linguistic factors. Psychological Research 50: 123-129.

Handley, S., Capon, A., Beveridge, M., Dennis, I. \& Evans, J. St. B.T. (2004). Working memory, inhibitory control, and the development of children's reasoning. Thinking and Reasoning 10: 175-195.

Hill, E.L. (2004). Executive disfunction in autism. Trends in Cognitive Sciences 8 (1): 26-32.

Inglis, M. \& Simpson, A. (2006). Characterising mathematical reasoning: studies with the Wason Selection Task. En Bosch, M. (ed). Proceedings of the Fourth Congress of the European Society for Research in Mathematics Education (pp. 1768-1777). Sant Feliu de Guíxols.

Kahneman, D. \& Tversky, A. (1973). On the psychology of prediction. Psychological Review 80: 237-251.

López Astorga, M. (2009). El problema de Linda y la falacia de la conjunción. Konvergencias. Filosofía y Culturas en Diálogo VII (21): 24-43.

López Astorga, M. (2009b). ¿Funciona el cerebro de los grandes maestros del ajedrez de manera diferente al de la población general? Ciencia Cognitiva 3 (3): 83-85.

Morsanyi, K., Handley, S.J. \& Evans, J. St. B.T. (2009). Heuristics and biases in autism: less biased but not more logical. En Taatgen, N.A. y van Rijn, H. (eds.). Proceedings of the $31^{s t}$ Annual Conference of the Cognitive Science Society (pp. 75-80). Cognitive Science Society: Austin (Texas).

Pijnacker, J., Geurts, B., Van Lambalgen, M., Kan, C.C., Buitelaar, J.K. \& Hagoort, P. (2009). Defeasible reasoning in high-functioning adults with autism: evidence for impaired exceptionhandling. Neuropsychologia 47 (3): 644-651.

Reyna, V.F. (2004). How people make decisions that involve risk: a dual-processes approach. Currents Directions in Psychological Science 13: 60-66.

Stanovich, K.E. (1999). Who is rational? Studies of individual differences in reasoning. Erlbaum. Mahwah, Nueva Jersey.

Tversky, A. \& Kahneman, D. (1983). Extensional versus intuitive reasoning: the conjunction fallacy in probability judgement. Psychological Review 90: 293-315. 
